\title{
SECOND ORDER SPIRAL SPLINES
}

LYLE NOAKES

ABSTRACT. Second order spiral splines are $C^{2}$ unit-speed planar curves that can be used to interpolate a list $Y$ of $n+1$ points in $\mathbb{R}^{2}$ at times specified in some list $T$, where $n \geq 2$. Asymptotic methods are used to develop a fast algorithm, based on a pair of tridiagonal linear systems and standard software. The algorithm constructs a second order spiral spline interpolant for data that is convex and sufficiently finely sampled.

\section{INTRODUCTION}

Given a finite sequence $T$ of real numbers $T_{0}<T_{1}<\ldots<T_{n}$, a pair $V$ of vectors $V_{0}, V_{n} \in \mathbb{R}^{m}$ and a finite sequence $Y$ of points $Y_{0}, Y_{1}, \ldots, Y_{n} \in \mathbb{R}^{m}$ where $m \geq 1$, an interpolant of $(Y, V)$ at $T$ is defined to be a $C^{2}$ curve $y:\left[T_{0}, T_{n}\right] \rightarrow \mathbb{R}^{m}$ satisfying $y\left(T_{j}\right)=Y_{j}$ for $0 \leq j \leq n$, as well as the auxiliary conditions $y^{(1)}\left(T_{0}\right)=V_{0}$ and $y^{(1)}\left(T_{n}\right)=V_{n}$ on derivatives $y^{(1)}$ of $y$ at $T_{0}$ and $T_{n}$. Then $y$ is said to interpolate $(Y, V)$ at $T$.

A standard interpolant is the minimiser $y$ of

$$
J(y):=\int_{T_{0}}^{T_{n}}\left\|y^{(2)}(t)\right\|^{2} d t
$$

where \|\| denotes the Euclidean norm, and $y^{(2)}$ is the second derivative. There is precisely one minimiser, namely the cubic polynomial spline which is the $C^{2}$ piecewise-cubic polynomial interpolant with knots at $T$. The cubic polynomial spline is easily calculated from $T$ and $(Y, V)$, by solving a tridiagonal system of linear equations [10. It is much more difficult, both from a theoretical and practical point of view, to find $C^{2}$ interpolants that are unit-speed, namely $\left\|y^{(1)}(t)\right\|=1$ for all $t \in\left[T_{0}, T_{n}\right]$.

Unit-speed curves are significant for highway and railway design [19], [20, motion planning for robots [14], and path planning for unmanned aerial vehicles and military aircraft 9], 18. Whereas cubic polynomial splines always exist, an evident necessary condition for existence of a $C^{2}$ unit-speed interpolant is

$$
\left\|Y_{j}-Y_{j-1}\right\| \leq L_{j}:=T_{j}-T_{j-1} \text { for } 1 \leq j \leq n .
$$

By an elastic spline we mean ${ }^{1}$ a critical point of the restriction of $J$ to the space of unit-speed interpolants. An elastic spline is known to be precisely a $C^{2}$ interpolant that is a track-sum $y$ of elastica (elastic curves) $y_{j}:\left[T_{j-1}, T_{j}\right] \rightarrow \mathbb{R}^{m}$. An elastica is defined as 2 a critical point of $J$ restricted to unit-speed curves $y_{j}$ where $y_{j}\left(T_{j-1}\right), y_{j}^{(1)}\left(T_{j-1}\right), y_{j}\left(T_{j}\right), y_{j}^{(1)}\left(T_{j}\right)$ are all prescribed in advance. Elastic splines are extensively studied, with well-developed algorithms for interpolation, as discussed in $\$ 2$. These algorithms for elastic splines require solutions of systems of nonlinear equations for variable parameters, and consequently very significant effort, compared with the sparse linear systems needed for interpolation by (non-unit-speed) polynomial splines.

Besides elastica there are simpler classes of unit-speed track-summands called polynomial spirals [14]. The well-studied class of clothoidal splines, whose curvatures are $C^{0}$ and piecewise-affine, is insufficiently rich for interpolation when $T$ is given in advance. In the present paper we suppose that the inequalities (1) hold strictly, and consider the larger class of second order spiral splines, namely interpolants that are $C^{2}$ track-sums $y_{\theta}$ of second order generalised Cornu spirals, whose curvatures are $C^{0}$ and piecewise-quadratic in the parameter $t$.

In the present paper the data $(Y, V)$ is generated by unknown strictly convex ${ }^{3}$ curves $x$ with suitably bounded derivatives, and sufficiently fine sampling specified by $T$. The setting is described in detail in $\$ 3$. In $\$ 4$, $\$ 5$ a pair of tridiagonal linear systems defines a $C^{1}$ cubic polynomial spline $\hat{\theta}:\left[t_{0}, T_{n}\right] \rightarrow \mathbb{R}$. Our construction echoes the well-known algorithm for polynomial cubic splines, where there is a single tridiagonal system [10. As with the classical algorithm, our linear systems are well-conditioned and very quick to solve. Our construction is more than twice as complicated as the classical algorithm, and requires more care to implement, but the description in $\$ 4$ \&5 is straightforward.

Unfortunately the same cannot be said for the proof of Theorem 1, which is our main result. The proof, spanning $\$ 6$, $\$ 7,88$ $\$ 9$ uses a number of difficult asymptotic expansions, followed by a sequence of careful manipulations and many equations. Theorem 1 says that, relative to a parameter $\epsilon>0$ measuring distances between successive data, we have $\hat{\theta}=\theta+O\left(\epsilon^{4}\right)$ where $y_{\theta}$ is the desired interpolant. This immediately gives rise to some approximate interpolants by spiral splines, as illustrated in Examples 1, 2, 3, More significantly, as demonstrated later in \$10, availability of a suitable initial guess is absolutely key to the nonlinear problem of determining $\theta$ and thereby $y_{\theta}$. The principle is illustrated in Examples 4, 5, 6.

Date: January 10, 2019.

${ }^{1}$ The term elastic spline is sometimes used differently, to mean an interpolant where $T$ is not specified in advance [5].

${ }^{2}$ The length of $y_{j}$ is then $T_{j}-T_{j-1}$. Sometimes the term elastica has a different meaning, where the length of $y_{j}$ is not prescribed in advance 5]. It seems that the plural of elastica is elastica.

${ }^{3}$ The nonconvex case is more complicated, with potentially $2^{n}$ interpolants. 


\section{More Background on Unit-Speed Interpolants}

A natural class of unit-speed interpolants is that of the unit-speed reparameterised polynomial splines, studied in [24, [11, 21 where there are algorithms for efficient computation. Whereas a polynomial spline can be found to interpolate $(Y, V)$ at $T$, the unit-speed reparameterisation interpolates $(Y, V)$ at a different set of parameter values. This does not work well when $T$ is given in advance, as in our situation. Similar difficulties arise with clothoidal splines] 23], 44, 3], [19, 8] and their $C^{1}$ generalisations [18]. The algorithms in these papers are of significant interest, but do not apply when $T$ is already prescribed.

Interpolation by unit-speed curves can also be performed using elastic splines [16, [12], namely ${ }^{5}$ critical points of the restriction of the functional $J$ to the space of unit-speed $C^{2}$ interpolants. An elastic spline turns out to be a $C^{2}$ track-sum $y$ of elastica $y_{j}:\left[T_{j-1}, T_{j}\right] \rightarrow \mathbb{R}^{m}$, satisfying the auxiliary end conditions. Elastica (elastic curves) have a long and interesting history [15], [5, [1, and their study reduces $s^{6}$ to the case where $m=3$. The elastica are completely known in terms of elliptic functions [22], with simplifications for $m=2$, which is the case of interest for the present paper. Although elastic splines (sometimes called nonlinear splines or true splines) are highly regarded7 as interpolants, they are less widely used than cubic polynomial splines. This is because the interpolation conditions for elastic splines require the solution of a system of nonlinear equations that is even more complicated ${ }^{8}$ than for clothoidal splines. However, the relative sophistication of elastica compared with cubic polynomials is not the main difficulty in computing elastic splines. As with most nonlinear problems, indeed also with our present task of finding spiral spline interpolants, the main difficulty is the construction of a suitable initial guess.

The algorithms in [16], [12] and [13] are for sliding elastic splines, where $T$ is not given in advance. Another condition that is sometimes imposed is that the total time $T_{n}-T_{0}$ should be fixed or, equivalently, the length of the spline is prescribed in advance. In the present paper the entire sequence $T$ is assumed to be given in advance, by analogy with standard interpolation by cubic polynomial splines.

What is different from cubic polynomial splines is that our interpolants are required to be unit-speed. Because of this additional requirement, once $T_{0}$ is given, fixing the rest of the sequence $T$ is equivalent to prescribing the lengths $L_{j}$ of the interpolant between all consecutive data points. In these circumstances, the main contribution to interpolation by elastic splines is Theorem 4.1 of [17, where a nonlinear system of equations is given for determining elastic splines in great generality. As usual with nonlinear problems, an initial guess is required in order to get started. Unfortunately [17] says very little about how this might be constructed. The present paper contributes something in this respect, although our focus is primarily on spiral splines, whereas [17] is concerned with elastic splines. Our guess hinges on the construction of $\hat{\theta}$ in $\S 4$, \& 5 , In $\$ 10$ standard software is used to improve $\hat{\theta}$ to $\theta$.

We also mention the discrete elastic splines of [6], where a discrete analogue of $J$ is optimised with respect to a variable finite sequence of points approximating $y$. In effect optimisation of $J$ with respect to $y$ is replaced by a large finite-dimensional optimisation, whose outcome depends critically on an unspecified initial guess in a high dimensional space.

In summary, reparameterised polynomial splines and clothoidal splines are unsuitable for interpolating $(Y, V)$ by $T$. Elastic splines come with non-negligible computational issues, especially the generation of suitable initial guesses.

\section{Convex Generators and Admissible Samplings}

Let $n \geq 2$ be given, together with $T_{0}<T_{n}, 0<C<B_{2}$, and $B_{i}$ where $3 \leq i \leq 5$. A unit speed $C^{\infty}$ curve $x:\left[T_{0}, T_{n}\right] \rightarrow \mathbb{R}^{2}$ is called a convex generator when $C<\left\|x^{(2)}\right\|_{\infty} \leq B_{2}$ and $\left\|x^{(i)}\right\|_{\infty} \leq B_{i}$ for $i=3,4$, 5. For brevity we write $X$ for the space $X_{T_{0}, T_{n}, C, B_{2}, B_{3}, B_{4}, B_{5}}$ of all convex generators.

Let $0<A_{m}<A_{M}$ be given, together with $\epsilon>0$ chosen small in comparison with the constants $C, B_{3}, B_{4}, B_{5}$ that define $X$. We define $\mathcal{T}_{\epsilon}$ to be the set of finite sequences $T$ of reals $T_{0}<T_{1}<T_{2}<\ldots<T_{n} \leq b$ such that $A_{m} \epsilon<L_{j}<A_{M} \epsilon$ for all $1 \leq j \leq n$.

A pair $(Y, V)$ is called admissibl $\S^{9}$ with respect to $T \in \mathcal{T}_{\epsilon}$ when $Y=\left(x\left(T_{0}\right), x\left(T_{1}\right), \ldots, x\left(T_{n}\right)\right)$ and $V=\left(x^{(1)}\left(T_{0}\right), x^{(1}\right)\left(T_{n}\right)$ for some convex generator $x \in X$. Then, for $1 \leq j \leq n$, set $q_{j}:=\left(Y_{j}-Y_{j-1}\right) / L_{j}, r_{j}:=\left\|q_{j}\right\|$, and

$$
k_{j}:=\frac{\sqrt{12\left(1-r_{j}^{2}\right)}}{L_{j} r_{j}} .
$$

Lemma 1. For small $\epsilon>0, r_{j}$ is bounded away from 0 , and $k_{j} \geq C+O(\epsilon)$ for all $1 \leq j \leq n$.

Proof: We have $x\left(T_{j}\right)-x\left(T_{j-1}\right)=x^{(1)}\left(T_{j-1}\right) L_{j}+x^{(2)}\left(T_{j-1}\right) \frac{L_{j}^{2}}{2}+x^{(3)}\left(T_{j-1}\right) \frac{L_{j}^{3}}{6}+O\left(\epsilon^{4}\right)$ where $x \in X$. In particular $L_{j} r_{j}=$ $1+O(\epsilon)$, and $r_{j}$ is bounded away from 0 when $\epsilon>0$ is small.

\footnotetext{
${ }^{4}$ A clothoidal spline is a unit-speed $C^{2}$ planar curve $t \mapsto y(t)$ whose curvature is $C^{0}$ and piecewise-affine in $t$.

${ }^{5}$ Elastic splines and elastica mean different things depending on the context 17. In the present paper, elastic splines are pinned and clamped, and elastica are fixed-length. In [16, $T$ is not given in advance and the elastic splines are sliding.

${ }^{6}$ On the other hand, the study of elastic splines does not reduce in this way.

${ }^{7}$ The objection raised at the end of [16] does not really apply when $T$ is given.

${ }^{8}$ As noted on p.184 of [12], clothoidal splines are sometimes used to construct initial guesses for the computation of elastic splines.

${ }^{9}$ The convexity assumption built into admissibility is the reason that we are able to find a unique estimate $\hat{\theta}$ (otherwise there would be many).
} 
Because $\left\|x^{(1)}\right\|=1$, we also have $\left\langle x^{(1)}, x^{(2)}\right\rangle=0$ and $\left\langle x^{(1)}, x^{(3)}\right\rangle=-\left\|x^{(2)}\right\|^{2}$, where $\langle$,$\rangle is the Euclidean inner product.$ Therefore, and by convexity,

$$
r_{j}^{2} L_{j}^{2}=L_{j}^{2}-\left\|x^{(2)}\left(T_{j-1}\right)\right\|^{2} \frac{L_{j}^{4}}{12}+O\left(\epsilon^{5}\right) \Longrightarrow 1-r_{j}^{2}=\left\|x^{(2)}\left(T_{j-1}\right)\right\|^{2} \frac{L_{j}^{2}}{12}+O\left(\epsilon^{3}\right) \geq \frac{C^{2} L_{j}^{2}}{12}+O\left(\epsilon^{3}\right) .
$$

So for small $\epsilon, 0<r_{j}<1$ and $k_{j}^{2}>12\left(1-r_{j}^{2}\right) / L_{j}^{2} \geq C^{2}+O(\epsilon)$.

From admissibility 10 the $Y_{j}$ are separated by no more than $A_{M} \epsilon$, and lie in the closed disc of radius $n A_{M} \epsilon$ centred on $Y_{0}$. Assuming $\epsilon$ is sufficiently small for the conclusion of Lemma 1 to hold, write $q_{j}=r_{j}\left(\cos \omega_{j}\right.$, $\left.\sin \omega_{j}\right)$. We also require $\omega_{1} \in$ $(-\pi, \pi)$, and $\omega_{j}=\omega_{j-1}+O(\epsilon)$ for $2 \leq j \leq n$, so that the $\omega_{j}$ are uniquely determined. By admissibility, $V_{0}=\left(\cos \nu_{0}, \sin \nu_{0}\right)$ and $V_{n}=\left(\cos \nu_{n}, \sin \nu_{n}\right)$ where $\nu_{0}=\omega_{1}+O(\epsilon)$ and $\nu_{n}=\omega_{n}+O(\epsilon)$.

Let $\theta:\left[T_{0}, T_{n}\right] \rightarrow \mathbb{R}$ be a $C^{1}$ cubic spline with knots $T_{j}$ such that $\theta\left(T_{0}\right)=\nu_{0}$ and $\theta\left(T_{n}\right)=\nu_{n}$. For $1 \leq j \leq n$ and all $s \in\left[0, L_{j}\right]$ write $\theta_{j}(s):=\theta\left(T_{j-1}+s\right)=a_{j}+b_{j} s+c_{j} s^{2}+d_{j} s^{3}$. Besides the two auxiliary end conditions,

$$
a_{1}=\nu_{0}, \quad a_{n}+b_{n} L_{n}+c_{n} L_{n}^{2}+d_{n} L_{n}^{3}=\nu_{n},
$$

there are $2 n-2$ conditions for $\theta$ to be $C^{1}$, namely

$$
\begin{aligned}
a_{j+1} & =a_{j}+b_{j} L_{j}+c_{j} L_{j}^{2}+d_{j} L_{j}^{3}, \\
b_{j+1} & =b_{j}+2 c_{j} L_{j}+3 d_{j} L_{j}^{2},
\end{aligned}
$$

where $1 \leq j \leq n-1$. So we have $2 n$ affine equality constraints on $4 n$ coefficients $a_{j}, b_{j}, c_{j}, d_{j}$. The $C^{1}$ spline $\theta$ determines a second order spiral spline $y_{\theta}:\left[T_{0}, T_{n}\right] \rightarrow \mathbb{R}^{2}$ given by

$$
y_{\theta}(t):=Y_{0}+\int_{T_{0}}^{t}(\cos \theta(s), \sin \theta(s)) d s .
$$

We see that $y_{\theta}$ is unit-speed with $y^{(1)}\left(T_{0}\right)=V_{0}$ and $y^{(1)}\left(T_{n}\right)=V_{n}$. We are interested in finding $\theta$ such that $y_{\theta}$ is an interpolant of $(Y, V)$ at $T$, namely for $1 \leq j \leq n$,

$$
\int_{0}^{L_{j}}\left(\cos \theta_{j}(s), \sin \theta_{j}(s)\right) d s=Y_{j}-Y_{j-1} \text {. }
$$

Condition (6) amounts to $2 n$ non-affine conditions on the $4 n$ coefficients, making $4 n$ conditions in total.

\section{Tridiagonal Systems}

Given $(Y, V)$ admissible with respect to $T \in \mathcal{T}_{\epsilon}$ where $\epsilon>0$ is small, form the $n \times n$ tridiagonal matrix

$$
\hat{\mathbf{T}}:=\left[\begin{array}{ccccccccc}
3 L_{1} & -L_{1} & 0 & 0 & 0 & 0 & 0 & \ldots & 0 \\
-L_{1} & 3\left(L_{1}+L_{2}\right) & -L_{2} & 0 & 0 & 0 & 0 & \ldots & 0 \\
0 & -L_{2} & 3\left(L_{2}+L_{3}\right) & -L_{3} & 0 & 0 & 0 & \ldots & 0 \\
0 & 0 & -L_{3} & 3\left(L_{3}+L_{4}\right) & -L_{4} & 0 & 0 & \ldots & 0 \\
\vdots & \vdots & \vdots & \vdots & \vdots & \vdots & \vdots & \vdots & \\
\vdots & \vdots & \vdots & \vdots & \vdots & \vdots & \vdots & \vdots & -L_{n-1} \\
0 & 0 & 0 & 0 & 0 & 0 & -L_{n-2} & 3\left(L_{n-2}+L_{n-1}\right) & 9 L_{n-1}+8 L_{n}
\end{array}\right]
$$

and, if $n \geq 3$, the tridiagonal matrix

$$
\tilde{\mathbf{T}}:=\left[\begin{array}{ccccccccc}
2 L_{1} & L_{1} & 0 & 0 & 0 & 0 & 0 & \ldots & 0 \\
L_{1} & 2\left(L_{1}+L_{2}\right) & L_{2} & 0 & 0 & 0 & 0 & \ldots & 0 \\
0 & L_{2} & 2\left(L_{2}+L_{3}\right) & L_{3} & 0 & 0 & 0 & \ldots & 0 \\
0 & 0 & L_{3} & 2\left(L_{3}+L_{4}\right) & L_{4} & 0 & 0 & \ldots & \\
\vdots & \vdots & \vdots & \vdots & \vdots & \vdots & \vdots & \vdots & \\
\vdots & \vdots & \vdots & \vdots & \vdots & \vdots & \vdots & \vdots & \\
0 & 0 & 0 & 0 & 0 & 0 & L_{n-2} & 2\left(L_{n-2}+L_{n-1}\right) & L_{n-1} \\
0 & 0 & 0 & 0 & 0 & 0 & 0 & L_{n-1} & 2 L_{n-1}+3 L_{n} / 2
\end{array}\right] .
$$

\footnotetext{
${ }^{10}$ Looking forward, admissibility is needed to guarantee the conclusions of Theorem 1 but may be troublesome to check in applications. In such cases (including the examples of the present paper) Theorem 1 may be taken to assert that when its conclusions are false, the sampling of $x$ is too irregular or too sparse. The cure, which seems to be rarely needed, is to sample more regularly and more often from $x$.
} 
Solve

$$
\tilde{\mathbf{T}} \tilde{b}=\tilde{\mathbf{R}}:=6\left[\begin{array}{c}
\omega_{1}-\nu_{0} \\
\omega_{2}-\omega_{1} \\
\omega_{3}-\omega_{2} \\
\omega_{4}-\omega_{3} \\
\vdots \\
\vdots \\
\omega_{n-1}-\omega_{n-2} \\
\left(3 \omega_{n}-\nu_{n}\right) / 2-\omega_{n-1}
\end{array}\right]
$$

for an $n$-dimensional column vector $\tilde{b}$.

Taking $\sigma$ to be the sign of the determinant of the $2 \times 2$ matrix $\left[V_{0} \vdots Y_{1}-Y_{0}\right]$, set

$$
\begin{aligned}
& \rho_{j}:=\sigma k_{j} \sqrt{1-\frac{k_{j}^{2} L_{j}^{2}}{20}-\frac{\left(\tilde{b}_{j+1}-\tilde{b}_{j}\right)^{2}}{60 k_{j}^{2}}} \text { for } 1 \leq j \leq n-1, \quad \text { and } \\
& \rho_{n}:=\sigma k_{n} \sqrt{1-\frac{k_{n}^{2} L_{n}^{2}}{20}-\frac{9}{60 k_{n}^{2}}\left(\frac{\omega_{n}-\nu_{n}}{L_{n}}+\frac{\tilde{b}_{n}}{2}\right)^{2}} .
\end{aligned}
$$

Then solve

$$
\hat{\mathbf{T}} \hat{b}=\left[\begin{array}{c}
24\left(\omega_{1}-\nu_{0}\right)-10 L_{1} \rho_{1} \\
24\left(\omega_{2}-\omega_{1}\right)-10\left(L_{1} \rho_{1}+L_{2} \rho_{2}\right) \\
24\left(\omega_{3}-\omega_{2}\right)-10\left(L_{2} \rho_{2}+L_{3} \rho_{3}\right) \\
24\left(\omega_{4}-\omega_{3}\right)-10\left(L_{3} \rho_{3}+L_{4} \rho_{4}\right) \\
\vdots \\
\vdots \\
24\left(\omega_{n-1}-\omega_{n-2}\right)-10\left(L_{n-2} \rho_{n-2}+L_{n-1} \rho_{n-1}\right) \\
24\left(2 \omega_{n}+\nu_{n}-3 \omega_{n-1}\right)-10\left(3 L_{n-1} \rho_{n-1}+4 L_{n} \rho_{n}\right)
\end{array}\right]
$$

for an $n$-dimensional column vector $\hat{b}$. Set

$$
\begin{aligned}
& \hat{c}_{j}:=\frac{-7 \hat{b}_{j}-3 \hat{b}_{j+1}}{4 L_{j}}+\frac{5 \rho_{j}}{2 L_{j}} \text { for } 1 \leq j \leq n-1, \\
& \hat{d}_{j}:=\frac{5\left(\hat{b}_{j}+\hat{b}_{j+1}\right)}{6 L_{j}^{2}}-\frac{5 \rho_{j}}{3 L_{j}^{2}} \quad \text { for } 1 \leq j \leq n-1, \\
& \hat{c}_{n}:=\frac{6\left(\omega_{n}-\nu_{n}\right)}{L_{n}^{2}}-\frac{2 \hat{b}_{n}}{L_{n}}+\frac{5 \rho_{n}}{L_{n}}, \\
& \hat{d}_{n}:=-\frac{20\left(\omega_{n}-\nu_{n}\right)}{3 L_{n}^{3}}+\frac{10 \hat{b}_{n}}{9 L_{n}^{2}}-\frac{40 \rho_{n}}{9 L_{n}^{2}},
\end{aligned}
$$

and finally $\hat{a}_{1}:=\nu_{0}$ with

$$
\hat{a}_{j+1}:=\hat{a}_{j}+\hat{b}_{j} L_{j}+\hat{c}_{j} L_{j}^{2}+\hat{d}_{j} L_{j}^{3} \quad \text { for } 1 \leq j \leq n-1 .
$$

Then, for all $1 \leq j \leq n$, define $\hat{\theta}_{j}:\left[0, L_{j}\right] \rightarrow \mathbb{R}$ by $\hat{\theta}_{j}(s):=\hat{a}_{j}+\hat{b}_{j} s+\hat{c}_{j} s^{2}+\hat{d}_{j} s^{3}$. A $C^{1}$ cubic polynomial spline $\hat{\theta}:\left[T_{0}, T_{n}\right] \rightarrow \mathbb{R}$ is then given by $\hat{\theta}\left(T_{n}\right):=\hat{\theta}_{n}\left(L_{n}\right)$, and $\hat{\theta}(t):=\hat{\theta}_{j}\left(t-T_{j-1}\right)$ for $t \in\left[T_{j-1}, T_{j}\right)$.

Theorem 1. $\hat{\theta}\left(T_{0}\right)=\nu_{0}, \hat{\theta}\left(T_{n}\right)=\nu_{n}$ and, for small $\epsilon>0,\|\theta-\hat{\theta}\|_{\infty}=O\left(\epsilon^{4}\right)$.

Corollary 1. $y_{\hat{\theta}}$ satisfies the auxiliary conditions (2), and $\left\|y_{\theta}-y_{\hat{\theta}}\right\|_{\infty}=O\left(\epsilon^{5}\right)$.

To prove Theorem 1 it suffices to show that, for all $1 \leq j \leq n$

$$
\begin{aligned}
a_{j} & =\hat{a}_{j}+O\left(\epsilon^{4}\right) \\
b_{j} & =\hat{b}_{j}+O\left(\epsilon^{3}\right) \\
c_{j} & =\hat{c}_{j}+O\left(\epsilon^{2}\right) \\
d_{j} & =\hat{d}_{j}+O(\epsilon) .
\end{aligned}
$$

We now prove some asymptotic lemmas, for use in $\$ 7,98$ where $(17)$ is proved in two steps. In $\$ 9$ it turns out that $(17)$ is the key ingredient for an otherwise easy proof of $(16),(18),(19)$, and this completes the proof of Theorem 1 . 
5. Approximation and Interpolation

By Corollary 1 , the $C^{2}$ second order spiral spline $y_{\hat{\theta}}$ satisfies $y_{\hat{\theta}}\left(T_{0}\right)=Y_{0}, y_{\hat{\theta}}^{(1)}\left(T_{0}\right)=V_{0}, y_{\hat{\theta}}^{(1)}\left(T_{n}\right)=V_{n}$ and $\left.y_{\hat{\theta}}\right)\left(T_{j}\right)=$ $Y_{j}+O\left(\epsilon^{5}\right)$ for $1 \leq j \leq n$. Usually $y_{\hat{\theta}}\left(T_{j}\right) \neq Y_{j}$ for $1 \leq j \leq n$. So $y_{\hat{\theta}}$ is only approximately an interpolant of $(Y, V)$.

Because of the way $y_{\theta}$ is constructed from $\theta$, interpolation errors at $T_{j}$ tend to accumulate as $j$ increases. This can be avoided by using $\theta$ differently to define $\bar{y}_{\theta}:\left[T_{0}, T_{j}\right] \rightarrow \mathbb{R}^{2}$ by $\bar{y}_{\theta}\left(T_{0}\right)=Y_{0}$ and for $1 \leq j \leq n$,

$$
\bar{y}_{\theta}(t):=Y_{j-1}+\int_{T_{j-1}}^{t}(\cos \theta(s), \sin \theta(s)) d s \quad \text { for } T_{j-1}<t<T_{j} .
$$

By construction $\bar{y}_{\theta}\left(T_{0}\right)=Y_{0}$, and $\bar{y}_{\theta}\left(T_{j}^{-}\right)=Y_{j}$ for all $j$, regardless of $\theta$. However unless $y_{\theta}$ interpolates $(Y, V)$ exactly at $T$ the left-interpolant $\bar{y}_{\theta}$ is not even a second order spiral spline, because $\bar{y}_{\theta}$ is only left-continuous but not continuous at $T_{j}$ for $1 \leq j \leq n-1$.

By Theorem $1, \bar{y}_{\hat{\theta}}\left(T_{j}\right)=Y_{j}+O\left(\epsilon^{5}\right)$ for $1 \leq j \leq n$, and $\bar{y}_{\hat{\theta}}^{(1)}\left(T_{0}\right)=V_{0}, \bar{y}_{\hat{\theta}}^{(1)}\left(T_{n}\right)=V_{n}$. So the left-interpolant $\bar{y}_{\hat{\theta}}$ approximately interpolates $(Y, V)$ at $T$. Because the errors in $\bar{y}_{\hat{\theta}}\left(T_{j}\right) \approx Y_{j}$ do not accumulate as $j$ increases, $\bar{y}_{\hat{\theta}}$ is better than $y_{\hat{\theta}}$ for diagnostics.

Example 1. Set $n=10$ with $T=(0,0.575,0.92,1.265,1.38,1.61,1.794,1.955,2.07,2.185,2.3)$, and let the convex generator be the second order spiral $x:[0,2.185] \rightarrow \mathbb{R}^{2}$ given by $x(0)=(0,0)$ and $x^{(1)}(t)=(\cos \psi(t)$, $\sin \psi(t))$ with $\psi(t)=t+t^{2}+t^{3}$. This generates dat ${ }^{11}(Y, V)$ where

$Y=((0,0),(0.494229,0.234572),(0.436814,0.544934),(0.196378,0.419176),(0.26536,0.333427),(0.387233,0.460597),(0.245648,0.44385)$,

$(0.348732,0.376214),(0.340667,0.473069),(0.262317,0.421501),(0.348435,0.395382))$

and $V=((1,0)),,(0.615762,0.787932))$. Computation of the $4 n$ coefficients for $\hat{\theta}$ took 0.000918 seconds in Mathematica on a 2015 a 2.2GHZ MacBook Air with 8 GB RAM. The plot of $y_{\hat{\theta}}$ is shown in Figure 1, together with the data. Although $\psi$ is just a cubic polynomial, the $C^{1}$ cubic spline $\hat{\theta}$ is not exactly $\psi$, as can be seen from the failures evident in Figure 1 of $y_{\hat{\theta}}$ to interpolate at $T_{3}, T_{4}, T_{6}, T_{9}$. However $y_{\hat{\theta}}$ is not a bad initial guess for an interpolant. Finer sampling would improve it. Coarser sampling would make it worse.

In Example 1 the convex generator $x$ is actually a second order spiral. Similar results are obtained when the data is generated by a more complicated convex curve.

Example 2. Take $T=(0,0.6,0.96,1.32,1.44,1.68,1.872,2.04,2.16,2.28,2.4)$ and let $x:[0,2.4] \rightarrow \mathbb{R}^{2}$ be given by $x(0)=$ $(0,0)$ and $x^{(1)}(t)=e^{t}+t(1+\sin (5 t) / 2)$. Using $x$ to generate data $(Y, V)$, Mathematica takes 0.000998 seconds to compute $\hat{\theta}$. The plot of $y_{\hat{\theta}}$ is shown in Figure 2, together with the data. In Figure 2, $y_{\hat{\theta}}$ almost interpolates the data, except near $T_{3}$, $T_{5}, T_{6}, T_{9}$

Example 3. Whereas in Example $2 y_{\hat{\theta}}$ is nearly acceptable, the quality of the approximate interpolant falls away very quickly if we use define $(Y, V)$ in the same way but over a larger interval $\left[T_{0}, T_{n}\right]$. Here we take $T=(0,0.675,1.08,1.485,1.62,1.89,2.106,2.295,2.43,2.565,2.7)$, and $x$ is given by the same formula over the larger interval $[0,2.7]$. Computation of $\hat{\theta}$ took 0.000986 seconds, and the plot of $y_{\hat{\theta}}$ is shown in Figure 3 , together with the data. We see that $y_{\hat{\theta}}$ is very far from interpolating, except at $T_{0}, T_{1}, T_{2}$. Even though the curve nearly passes through the terminal point $Y_{10}$, it arrives too soon and overshoots. This poor performance can be corrected by more refined sampling or, as illustrated in Example 6, by the more sophisticated method of $\$ 10$ which starts with $\hat{\theta}$.

\section{Some Asymptotic Lemmas}

Recall $\theta_{j}(s)=a_{j}+b_{j} s+c_{j} s^{2}+d_{j} s^{3}$ where $s \in\left[0, L_{j}\right]$ for $1 \leq j \leq n$, and $Y, V, T$ are given with $(Y, V)$ is admissible with respect to $T$. Using Mathematica to calculate the Taylor polynomial $\hat{F}_{j}(s)$ of degree 4 of $\left.F_{j}(s):=\int_{0}^{s}(\cos u, \sin u) d u\right)$ about $s=0$, we find

Lemma 2. For $1 \leq j \leq n$,

$$
\hat{F}_{j}\left(L_{j}\right)=L_{j}\left[\begin{array}{cc}
\cos a_{j} & -\sin a_{j} \\
\sin a_{j} & \cos a_{j}
\end{array}\right]\left[\begin{array}{c}
\alpha_{j} \\
\beta_{j}
\end{array}\right]
$$

where

$$
\alpha_{j}:=1-\frac{b_{j}^{2} L_{j}^{2}}{6}-\frac{b_{j} c_{j} L_{j}^{3}}{4}+\frac{\left(b_{j}^{4}-12 c_{j}^{2}-24 b_{j} d_{j}\right) L_{j}^{4}}{120} \text { and } \beta_{j}:=\frac{b_{j} L_{j}}{2}+\frac{c_{j} L_{j}^{2}}{3}-\frac{\left(b_{j}^{3}-6 d_{j}\right) L_{j}^{3}}{24}-\frac{b_{j}^{2} c_{j} L_{j}^{4}}{10} .
$$

From Lemma 2

$$
L_{j} r_{j}\left[\begin{array}{c}
\cos \omega_{j} \\
\sin \omega_{j}
\end{array}\right]=L_{j} q_{j}=Y_{j}-Y_{j-1}=F_{j}\left(L_{j}\right)=L_{j}\left[\begin{array}{cc}
\cos a_{j} & -\sin a_{j} \\
\sin a_{j} & \cos a_{j}
\end{array}\right]\left[\begin{array}{c}
\alpha_{j} \\
\beta_{j}
\end{array}\right]+O\left(\epsilon^{5}\right) \Longrightarrow
$$

\footnotetext{
${ }^{11}$ We have chosen $T$ in order to sample from $x$ somewhat more frequently where curvature is large. Finer sampling always helps.
} 


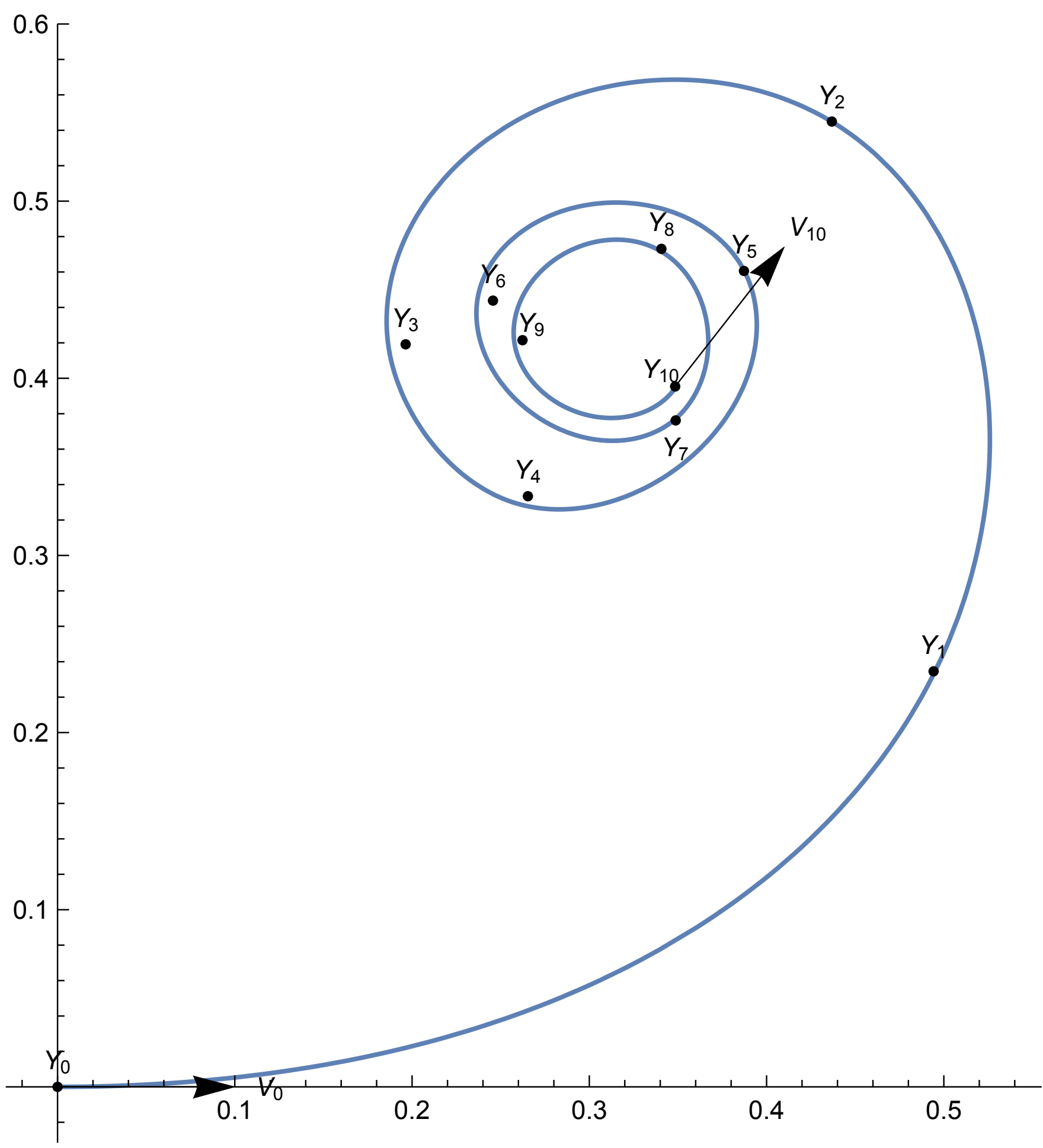

Figure 1. $y_{\hat{\theta}}$ for Example 1

$(20)$

$(21)$

$$
\begin{aligned}
r_{j}^{2} & =\alpha_{j}^{2}+\beta_{j}^{2}+O\left(\epsilon^{4}\right) \\
\left(\cos \left(a_{j}-\omega_{j}\right), \sin \left(a_{j}-\omega_{j}\right)\right) & =\frac{\left(\alpha_{j}, \beta_{j}\right)}{\left\|\left(\alpha_{j}, \beta_{j}\right)\right\|}+O\left(\epsilon^{5}\right) .
\end{aligned}
$$

Lemma 3.

$$
\begin{aligned}
& \left(b_{j}+c_{j} L_{j}+\frac{9}{10} d_{j} L_{j}^{2}\right)^{2}+\frac{c_{j}^{2} L_{j}^{2}}{15}=k_{j}^{2}\left(1-\frac{k_{j}^{2} L_{j}^{2}}{20}\right)+O\left(\epsilon^{3}\right) \quad \text { for } 1 \leq j \leq n, \\
& a_{j}+\frac{b_{j} L_{j}}{2}+\frac{c_{j} L_{j}^{2}}{3}+\frac{d_{j} L_{j}^{3}}{4}=\omega_{j}+O\left(\epsilon^{4}\right) \quad \text { for } 1 \leq j \leq n, \\
& \text { (24) } \frac{b_{j+1} L_{j+1}+b_{j} L_{j}}{2}+\frac{c_{j+1} L_{j+1}^{2}+2 c_{j} L_{j}^{2}}{3}+\frac{d_{j+1} L_{j+1}^{3}+3 d_{j} L_{j}^{3}}{4}=\omega_{j+1}-\omega_{j}+O\left(\epsilon^{4}\right) \quad \text { for } 1 \leq j \leq n-1 \text {. }
\end{aligned}
$$

Proof: Using Mathematica to substitute for $r_{j}^{2}$ from 20 in $k_{j}^{2}$ and then expand in powers of $L_{j}$, we obtain

$$
\left(b_{j}+c_{j} L_{j}+\frac{9}{10} d_{j} L_{j}^{2}\right)^{2}+\left(\frac{b_{j}^{4}}{20}+\frac{c_{j}^{2}}{15}\right) L_{j}^{2}=k_{j}^{2}
$$




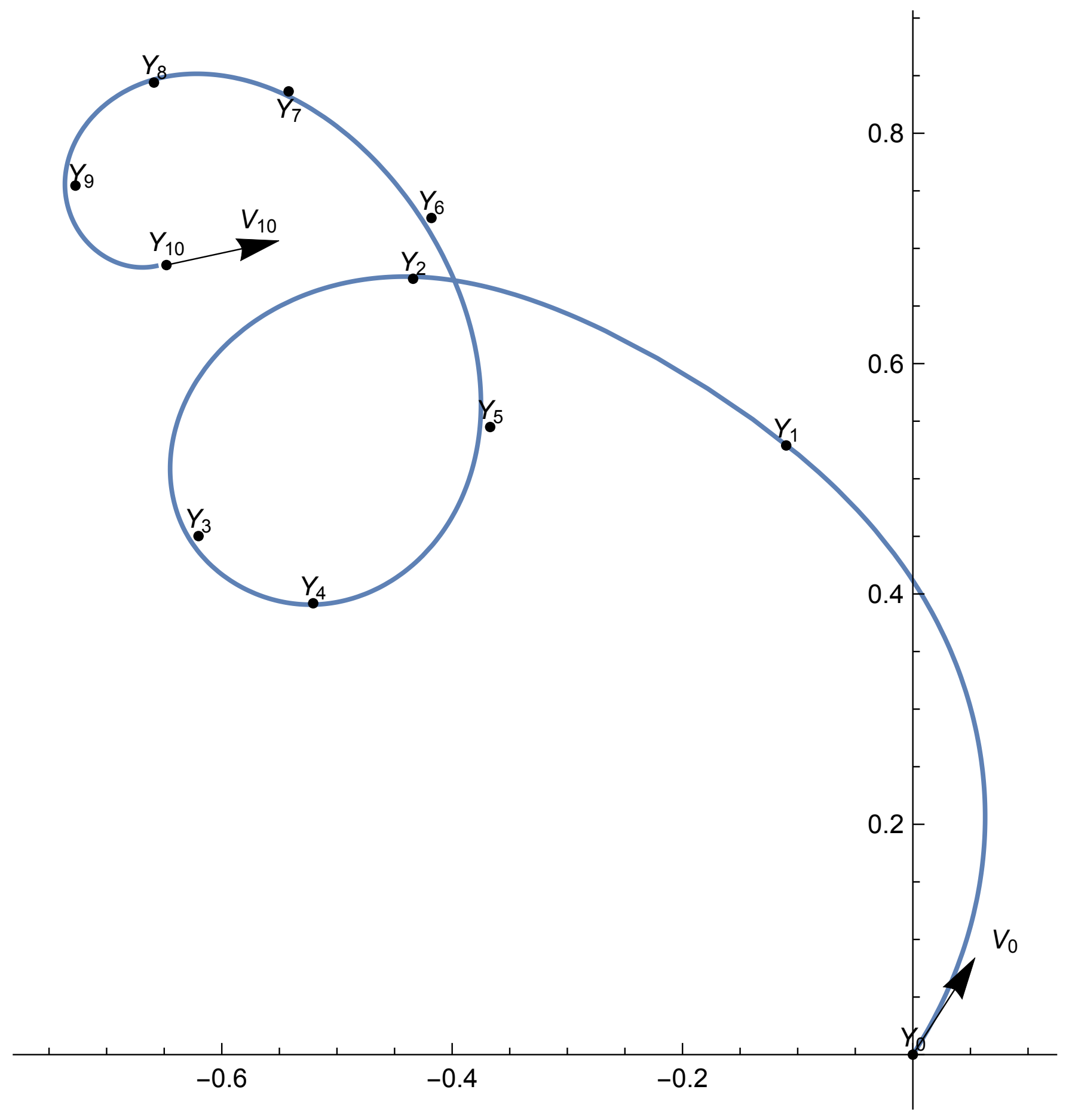

FIGURE 2. $y_{\hat{\theta}}$ for Example 2

where $1 \leq j \leq n$. In particular, $b_{j}^{2}=k_{j}^{2}+O(\epsilon)$. Substituting $b_{j}^{4}=k_{j}^{4}+O(\epsilon)$ back in 25 gives $(22)$. For $(23)$ use Mathematica to Taylor expand the argument of the right hand side of (21) in powers of $L_{j}$. For (24) substitute in (3) for $a_{j}, a_{j+1}$ from (23).

7. Estimating the $b_{j}$ To $O\left(\epsilon^{2}\right)$

From (24), for $1 \leq j \leq n-1$,

$$
\frac{b_{j+1} L_{j+1}+b_{j} L_{j}}{2}+\frac{c_{j+1} L_{j+1}^{2}+2 c_{j} L_{j}^{2}}{3}=\omega_{j+1}-\omega_{j}+O\left(\epsilon^{3}\right) .
$$

From (23) with $j=1$, and from (2),

$$
c_{1} L_{1}=\frac{3\left(\omega_{1}-\nu_{0}\right)}{L_{1}}-\frac{3 b_{1}}{2}+O\left(\epsilon^{2}\right)
$$

From (4) for $1 \leq j \leq n-1$,

$$
c_{j} L_{j}=\frac{b_{j+1}-b_{j}}{2}+O\left(\epsilon^{2}\right) .
$$

From (23) with $j=n$, and from (2),

(28) $\quad \omega_{n}-\frac{b_{n} L_{n}}{2}-\frac{c_{n} L_{n}^{2}}{3}=a_{n}+O\left(\epsilon^{3}\right)=\nu_{n}-b_{n} L_{n}-c_{n} L_{n}^{2} \Longrightarrow c_{n} L_{n}=-\frac{3\left(\omega_{n}-\nu_{n}\right)}{2 L_{n}}-\frac{3 b_{n}}{4}+O\left(\epsilon^{2}\right)$. 


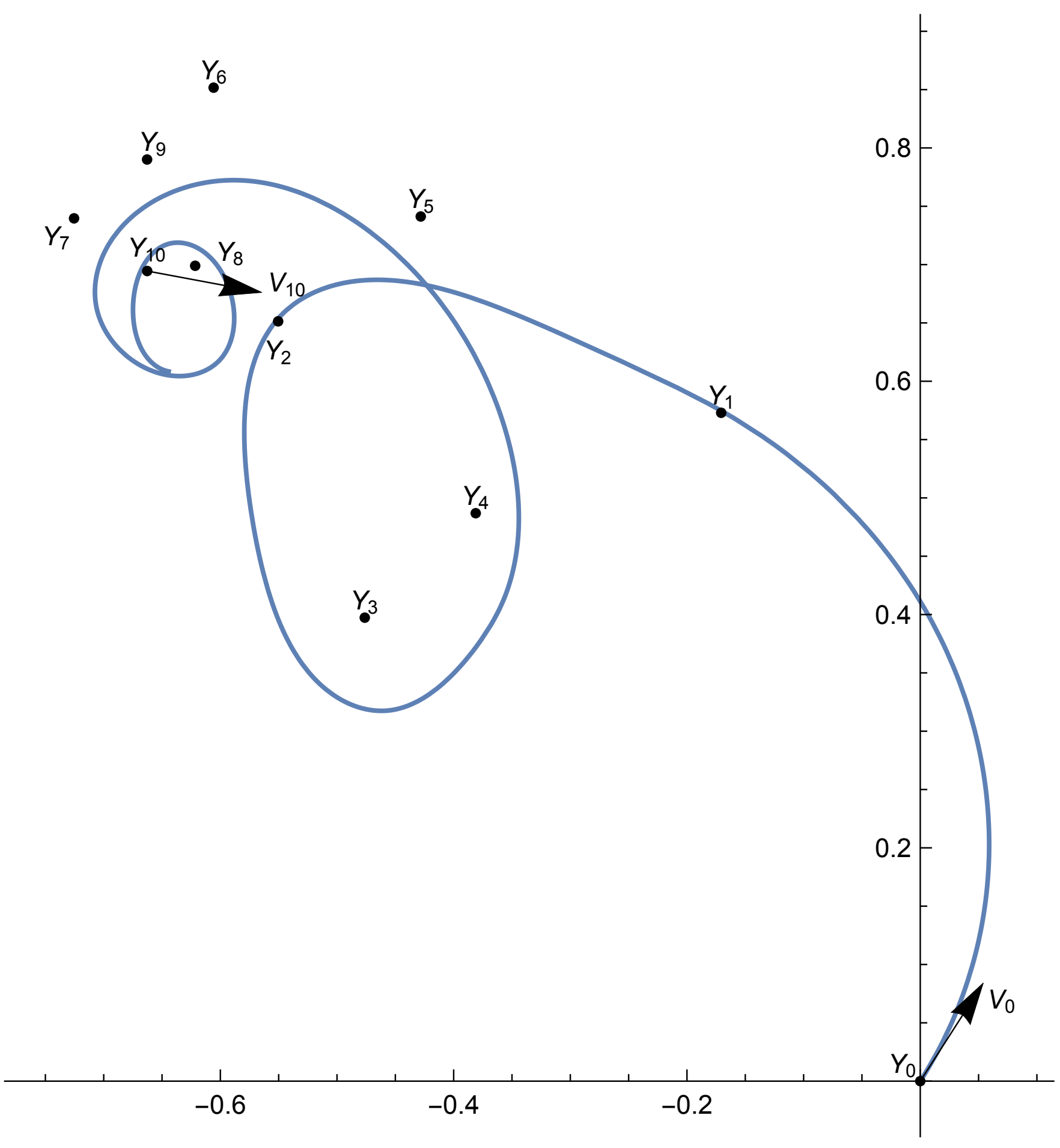

FiguRE 3. $y_{\hat{\theta}}$ for Example 3

Substituting for $c_{1} L_{1}$ in (27), and for $c_{1} L_{1}, c_{2} L_{2}, \ldots, c_{n} L_{n}$ in 26) with $1 \leq j \leq n-1$, we obtain the system

$$
\begin{aligned}
2 L_{1} b_{1}+L_{1} b_{2} & =6\left(\omega_{1}-\nu_{0}\right)+O\left(\epsilon^{3}\right), \\
L_{j-1} b_{j-1}+2\left(L_{j-1}+L_{j}\right) b_{j}+L_{j+1} b_{j+1} & =6\left(\omega_{j}-\omega_{j-1}\right)+O\left(\epsilon^{3}\right) \quad \text { for } 2 \leq j \leq n-2, \\
L_{n-1} b_{n-1}+\left(2 L_{n-1}+\frac{3 L_{n}}{2}\right) b_{n} & =3\left(3 \omega_{n}-\nu_{n}-2 \omega_{n-1}\right)+O\left(\epsilon^{3}\right),
\end{aligned}
$$

of $n$ linear equations for $b:=\left(b_{1}, b_{2}, \ldots, b_{n}\right)$, namely

(29)

$$
\tilde{\mathbf{T}} b=\tilde{\mathbf{R}}+O\left(\epsilon^{3}\right)=\tilde{\mathbf{T}} \tilde{b}+O\left(\epsilon^{3}\right)
$$

where $\tilde{\mathbf{T}}$ is the $n \times n$ tridiagonal matrix and $\tilde{\mathbf{R}}, \tilde{b}$ are the $n$-dimensional vectors all defined in $\$ 4$.

Lemma 4. $b=\tilde{b}+O\left(\epsilon^{2}\right)$.

Proof: Let $D_{\tilde{\mathbf{T}}}$ be the diagonal $n \times n$ matrix with the same diagonal entries as $\tilde{\mathbf{T}}$. Because $A_{m} \epsilon<L_{j}<A_{M} \epsilon$, we see that $\left\|D_{\tilde{\mathbf{T}}}^{-1}\right\|_{\infty} \leq 1 /\left(2 A_{m} \epsilon\right)$. By $\sqrt[29]{ }, \tilde{\mathbf{T}}(b-\tilde{b})=O\left(\epsilon^{3}\right)$, and therefore

$$
\left\|D_{\tilde{\mathbf{T}}}^{-1} \tilde{\mathbf{T}}(b-\tilde{b})\right\|=O\left(\epsilon^{2}\right) .
$$

Because $\tilde{\mathbf{T}}$ is strictly diagonally dominant by rows with dominance factor $1 / 2, D_{\tilde{\mathbf{T}}}^{-1} \tilde{\mathbf{T}}$ has condition number $\leq 3$. 
By (4) and Lemma 4,

$$
\text { 8. Proof of 17) }
$$

$$
c_{j} L_{j}=\frac{b_{j+1}-b_{j}}{2}+O\left(\epsilon^{2}\right)=\frac{\tilde{b}_{j+1}-\tilde{b}_{j}}{2}+O\left(\epsilon^{2}\right)
$$

for $1 \leq j \leq n-1$. Similarly by 28 and Lemma 4 . $c_{n} L_{n}=\frac{3\left(\omega_{n}-\nu_{n}\right)}{2 L_{n}}-\frac{3 \tilde{b}_{n}}{4}+O\left(\epsilon^{2}\right)$. Substituting for the $c_{j} L_{j}$ in 222 ,

$$
\begin{aligned}
&\left(b_{j}+c_{j} L_{j}+\frac{9}{10} d_{j} L_{j}^{2}\right)^{2}=k_{j}^{2}\left(1-\frac{k_{j}^{2} L_{j}^{2}}{20}\right)-\frac{\left(\tilde{b}_{j+1}-\tilde{b}_{j}\right)^{2}}{60}+O\left(\epsilon^{3}\right) \quad \text { for } 1 \leq j \leq n-1, \text { and } \\
&\left(b_{n}+c_{n} L_{n}+\frac{9}{10} d_{n} L_{n}^{2}\right)^{2}=k_{n}^{2}\left(1-\frac{k_{n}^{2} L_{n}^{2}}{20}\right)-\frac{9}{60}\left(\frac{\omega_{n}-\nu_{n}}{L_{n}}+\frac{\tilde{b}_{n}}{2}\right)^{2}+O\left(\epsilon^{3}\right),
\end{aligned}
$$

where the right hand sides are known to $O\left(\epsilon^{3}\right)$. By Lemma 1, for $\epsilon$ small the $k_{j}$ are bounded away from 0 for all $1 \leq j \leq n$. Therefore

$$
b_{j}+c_{j} L_{j}+\frac{9}{10} d_{j} L_{j}^{2}=\sigma_{j}\left|\rho_{j}\right|+O\left(\epsilon^{3}\right)=\sigma_{j} k_{j}+O\left(\epsilon^{2}\right)
$$

where $\sigma_{j}= \pm 1$, and the $\rho_{j}$ are defined as in (8), (9). In particular, by Lemma $1 \sigma_{j}=\operatorname{sign}\left(b_{j}\right)$ for $1 \leq j \leq n$. By (4), $b_{j}=b_{j-1}+O(\epsilon)$ for $2 \leq j \leq n$. Because the $k_{j}$ are bounded away from 0 so are the $\left|b_{j}\right|$. So the $\sigma_{j}$ are all equal. Recall the definition of $\sigma$ in $\$ 4$, namely $\sigma:=\operatorname{sign}\left(\operatorname{det}\left[V_{0} \vdots Y_{1}-Y_{0}\right]\right)$.

Lemma 5. For $\epsilon>0$ small, we have $\sigma_{j}=\sigma$ where $1 \leq j \leq n$.

Proof: By (4), $b_{1}=b_{2}+O(\epsilon)$. Then, from the first equation in system (7),

$$
3 b_{1}=2 b_{1}+b_{2}+O(\epsilon)=2 \tilde{b}_{1}+\tilde{b}_{2}+O(\epsilon)=\frac{6\left(\omega_{1}-\nu_{0}\right)}{L_{1}}+O(\epsilon) \Longrightarrow \sigma_{1}=\operatorname{sign}\left(\omega_{1}-\nu_{0}\right),
$$

where we use $\sigma_{1}=\operatorname{sign}\left(b_{1}\right)$. Now $V_{0}=x^{(1)}\left(T_{0}\right)$ and $Y_{j}=x\left(T_{j}\right)$ for $0 \leq j \leq n$, where $x:\left[T_{0}, T_{n}\right] \rightarrow \mathbb{R}^{2}$ is a convex generator. Write $x^{(1)}(t)=(\cos \psi(t), \sin \psi(t))$ where $\psi$ is $C^{1}$ and $\psi\left(T_{0}\right)=\nu_{0}$. By convexity, $\left|\psi^{(1)}\left(T_{0}\right)\right|=\left\|x^{(2)}\left(T_{0}\right)\right\|>C>0$. Taylor expanding $x$ to second order in $t$ about $T_{0}$, and recalling the definition of $\omega_{j}$,

$$
\begin{aligned}
& L_{1} r_{1}\left(\cos \omega_{1}, \sin \omega_{1}\right)=Y_{1}-Y_{0}=L_{1}\left(\cos \nu_{0}, \sin \nu_{0}\right)+\frac{L_{1}^{2}}{2} \psi^{(1)}\left(T_{0}\right)\left(-\sin \nu_{0}, \cos \nu_{0}\right)+O\left(\epsilon^{3}\right) \Longrightarrow \\
& L_{1} r_{1} \sin \left(\omega_{1}-\nu_{0}\right)=\frac{L_{1}^{2}}{2} \psi^{(1)}\left(T_{0}\right)+O\left(\epsilon^{3}\right)=\operatorname{det}\left[V_{0} \vdots Y_{1}-Y_{0}\right]+O\left(\epsilon^{3}\right) \Longrightarrow \operatorname{sign}\left(\omega_{1}-\nu_{0}\right)=\sigma .
\end{aligned}
$$

So $\sigma_{n}=\sigma_{n-1}=\ldots=\sigma_{1}=\sigma$.

From Lemma 5 we now have, for $1 \leq j \leq n$,

$$
b_{j}+c_{j} L_{j}+\frac{9}{10} d_{j} L_{j}^{2}=\rho_{j}+O\left(\epsilon^{3}\right)
$$

By (4), (30), for each $1 \leq j \leq n-1$,

$$
\begin{aligned}
2 c_{j} L_{j}+3 d_{j} L_{j}^{2} & =b_{j+1}-b_{j}+O\left(\epsilon^{3}\right), \\
c_{j} L_{j}+\frac{9}{10} d_{j} L_{j}^{2} & =\rho_{j}-b_{j}+O\left(\epsilon^{3}\right),
\end{aligned}
$$

where the right hand sides are presently known only with $O\left(\epsilon^{2}\right)$ errors. Nevertheless, solving these systems with $1 \leq j \leq n-1$,

$$
\begin{aligned}
& c_{j} L_{j}=\frac{-7 b_{j}-3 b_{j+1}}{4}+\frac{5 \rho_{j}}{2}+O\left(\epsilon^{3}\right), \\
& d_{j} L_{j}^{2}=\frac{5\left(b_{j}+b_{j+1}\right)}{6}-\frac{5 \rho_{j}}{3}+O\left(\epsilon^{3}\right) .
\end{aligned}
$$

By (23) with $j=n$ and (2), and by (30) with $j=n$,

Solving,

$$
\begin{aligned}
8 c_{n} L_{n}+9 d_{n} L_{n}^{2} & =\frac{12\left(\nu_{n}-\omega_{n}\right)}{L_{n}}-6 b_{n}+O\left(\epsilon^{3}\right), \\
10 c_{n} L_{n}+9 d_{n} L_{n}^{2} & =10 \rho_{n}-10 b_{n}+O\left(\epsilon^{3}\right) .
\end{aligned}
$$

$$
\begin{aligned}
c_{n} L_{n} & =\frac{6\left(\omega_{n}-\nu_{n}\right)}{L_{n}}-2 b_{n}+5 \rho_{n}+O\left(\epsilon^{3}\right), \\
d_{n} L_{n}^{2} & =-\frac{20\left(\omega_{n}-\nu_{n}\right)}{3 L_{n}}+\frac{10 b_{n}}{9}-\frac{40 \rho_{n}}{9}+O\left(\epsilon^{3}\right) .
\end{aligned}
$$

In particular, substituting for $c_{1} L_{1}, d_{1} L_{1}^{2}$ in 23 with $j=1$,

$$
3 L_{1} b_{1}-L_{1} b_{2}=24\left(\omega_{1}-\nu_{0}\right)-10 L_{1} \rho_{1}+O\left(\epsilon^{4}\right) .
$$

Substituting for $c_{j} L_{j}, d_{j} L_{j}^{2}$ where $1 \leq j \leq n$ in 24 , gives $n-1$ linear equations for the remaining components of $b$, namely 
$-L_{j} b_{j}+3\left(L_{j}+L_{j+1}\right) b_{j+1}-L_{j+1} b_{j+2}=24\left(\omega_{j+1}-\omega_{j}\right)-10\left(L_{j} \rho_{j}+L_{j+1} \rho_{j+1}\right)+O\left(\epsilon^{4}\right) \quad$ for $1 \leq j \leq n-2, \quad$ and $-3 L_{n-1} b_{n-1}+\left(9 L_{n-1}+8 L_{n}\right) b_{n}=24\left(2 \omega_{n}+\nu_{n}-3 \omega_{n-1}\right)-10\left(3 L_{n-1} \rho_{n-1}+4 L_{n} \rho_{n}\right)+O\left(\epsilon^{4}\right)$.

So $\hat{\mathbf{T}} b=\hat{R}+O\left(\epsilon^{4}\right)$ and 17 follows, in the same way as for Lemma 4 .

\section{Proof of Theorem 1}

As noted at the end of 44 , now that (17) is proved it suffices to verify (16), (18), (19). Using (17) in (31), (35), then in (33), (35),

$$
\begin{aligned}
& c_{j} L_{j}=\frac{-7 b_{j}-3 b_{j+1}}{4}+\frac{5 \rho_{j}}{2}+O\left(\epsilon^{3}\right)=\frac{-7 \hat{b}_{j}-3 \hat{b}_{j+1}}{4}+\frac{5 \rho_{j}}{2}+O\left(\epsilon^{3}\right)=\hat{c}_{j} L_{j}+O\left(\epsilon^{3}\right) \quad \text { for } 1 \leq j \leq n-1, \\
& d_{j} L_{j}^{2}=\frac{5\left(b_{j}+b_{j+1}\right)}{6}-\frac{5 \rho_{j}}{3}+O\left(\epsilon^{3}\right)=\frac{5\left(\hat{b}_{j}+\hat{b}_{j+1}\right)}{6}-\frac{5 \rho_{j}}{3}+O\left(\epsilon^{3}\right)=\hat{d}_{j} L_{j}^{2}+O\left(\epsilon^{3}\right) \quad \text { for } 1 \leq j \leq n-1, \\
& c_{n} L_{n}=\frac{6\left(\omega_{n}-\nu_{n}\right)}{L_{n}}-2 b_{n}+5 \rho_{n}+O\left(\epsilon^{3}\right)=\frac{6\left(\omega_{n}-\nu_{n}\right)}{L_{n}}-2 \hat{b}_{n}+5 \rho_{n}+O\left(\epsilon^{3}\right)=\hat{c}_{n} L_{n}+O\left(\epsilon^{3}\right), \\
& d_{n} L_{n}^{2}=-\frac{20\left(\omega_{n}-\nu_{n}\right)}{3 L_{n}}+\frac{10 b_{n}}{9}-\frac{40 \rho_{n}}{9}+O\left(\epsilon^{3}\right)=-\frac{20\left(\omega_{n}-\nu_{n}\right)}{3 L_{n}}+\frac{10 \hat{b}_{n}}{9}-\frac{40 \rho_{n}}{9}+O\left(\epsilon^{3}\right)=\hat{d}_{n} L_{n}^{2}+O\left(\epsilon^{3}\right),
\end{aligned}
$$

according to definitions (11), (12), (13), (14) in $\$ 4$. This proves (18), (19). Finally by (2) $a_{1}=\nu_{0}=\hat{a}_{1}$, and by (3) $a_{j+1}=a_{j}+b_{j} L_{j}+c_{j} L_{j}^{2}+d_{j} L_{j}^{3}=\hat{a}_{j}+b_{j} L_{j}+\hat{c}_{j} L_{j}^{2}+\hat{d}_{j} L_{j}^{3}+O\left(\epsilon^{4}\right)=\hat{a}_{j+1}+O\left(\epsilon^{4}\right)$ according to 15 for $1 \leq j \leq n-1$. So (16) is proved by induction.

\section{Closing Gaps}

As noted in $\$ 5$, the $C^{2}$ unit-speed curve $y_{\hat{\theta}}$ does not interpolate $(Y, V)$ exactly at $T$. Usually there are $O\left(\epsilon^{5}\right)$ errors in the interpolation conditions. The left-interpolant $\bar{y}_{\hat{\theta}}$ is not much better, except to illustrate the magnitudes and locations of errors, because $\bar{y}_{\hat{\theta}}$ is not even $C^{0}$.

When sampling is sufficiently fine, namely for sufficiently small $\epsilon>0$, the curves $y_{\hat{\theta}}$ and $\bar{y}_{\hat{\theta}}$ are almost indistinguishable, and either of these unit-speed approximate interpolants might be used in practice. This is already true for some parts of $y_{\hat{\theta}}$ in Example 1 and for most of $y_{\hat{\theta}}$ in Example 2. However $y_{\hat{\theta}}$ is seriously problematic in Example 3.

If finer sampling is inconvenient or impossible then, after finding $\hat{\theta}$, there is a second calculation we can do to correct the kinds of errors observed in Example 3, and also to a smaller extent in Examples 1, 2

We rewrite the conditions for a second order spiral spline interpolant as a nonlinear system of equations, then solve numerically using $\hat{\theta}$ to generate an initial guess. This is easier than finding $\hat{\theta}$ and simpler to code, but not so remarkably fast. It is unlikely to fail except when sampling is so coarse that $\hat{\theta}$ does not give a good initial guess. Even in the case of Example 3 the rough estimate $\hat{\theta}$ is enough to get started.

Given $(Y, V)$ admissible with respect to $T$, first calculate $\hat{\theta}$ as in $\S_{4}$. Then, for $u, v \in \mathbb{R}^{n}$, define $a_{j}, b_{j}, c_{j}, d_{j} \in \mathbb{R}$ for $1 \leq j \leq n$ by $c_{j}:=u_{j}$ and $d_{j}:=v_{j}$ for all $1 \leq j \leq n$, and $a_{1}:=\nu_{0}$. Using (3) and (4) $j-1$ times where $1 \leq j \leq n$,

$$
\begin{aligned}
& b_{j}=b_{1}+2 \sum_{k=1}^{j-1} u_{k} L_{k}+3 \sum_{k=1}^{j-1} v_{k} L_{k}^{2} \text { and } \\
& a_{j}=\nu_{0}+\sum_{k=1}^{j-1} b_{k} L_{k}+\sum_{k=1}^{j-1} u_{k} L_{k}^{2}+\sum_{k=1}^{j-1} v_{k} L_{k}^{3} .
\end{aligned}
$$

Using (35) to substitute for $b_{k}$ in (36),

$$
a_{j}=\nu_{0}+\left(T_{j-1}-T_{0}\right) b_{1}+2 \sum_{k=1}^{j-1} \sum_{i=1}^{k-1} u_{i} L_{i} L_{k}+3 \sum_{k=1}^{j-1} \sum_{i=1}^{k-1} v_{i} L_{i}^{2} L_{k}+\sum_{k=1}^{j-1} u_{k} L_{k}^{2}+\sum_{k=1}^{j-1} v_{k} L_{k}^{3}
$$

where $2 \leq j \leq n$. Similarly, using the second part of (2) in place of (3),

$$
\begin{aligned}
\nu_{n} & =\nu_{0}+\left(T_{n}-T_{0}\right) b_{1}+2 \sum_{k=1}^{n} \sum_{i=1}^{k-1} u_{i} L_{i} L_{k}+3 \sum_{k=1}^{n} \sum_{i=1}^{k-1} v_{i} L_{i}^{2} L_{k}+\sum_{k=1}^{n} u_{k} L_{k}^{2}+\sum_{k=1}^{n} v_{k} L_{k}^{3} \Longrightarrow \\
b_{1} & =\frac{\nu_{n}-\nu_{0}-2 \sum_{k=1}^{n} \sum_{i=1}^{k-1} u_{i} L_{i} L_{k}-3 \sum_{k=1}^{n} \sum_{i=1}^{k-1} v_{i} L_{i}^{2} L_{k}-\sum_{k=1}^{n} u_{k} L_{k}^{2}-\sum_{k=1}^{n} v_{k} L_{k}^{3}}{T_{n}-T_{0}} .
\end{aligned}
$$

Then $a_{j}$ and $b_{j}$ are also determined for $2 \leq j \leq n$, by substitution for $b_{1}$ in (37) and in (35). For $1 \leq j \leq n$ define $\theta_{j}:\left[0, L_{j}\right] \rightarrow \mathbb{R}$ by $\theta_{j}(t):=a_{j}+b_{j} t+c_{j} t^{2}+d_{j} t^{3}$. Define $\theta:\left[T_{0}, T_{n}\right] \rightarrow \mathbb{R}$ by $\theta\left(T_{0}\right):=\nu_{0}$ and $\theta(t):=\theta_{j}\left(t-T_{j-1}\right)$ for $t \in\left(T_{j-1}, T_{j}\right]$. Because the $a_{j}, b_{j}, c_{j}, d_{j}$ satisfy (3), (4), as well as the auxiliary conditions (2), the following lemma is easily verified.

Lemma 6. $\theta:\left[T_{0}, T_{n}\right] \rightarrow \mathbb{R}$ is a cubic polynomial spline with $\theta\left(T_{0}\right)=\nu_{0}$ and $\theta\left(T_{n}\right)=\nu_{n}$. 
So for any $u, v \in \mathbb{R}^{n}, y_{\theta}:\left[T_{0}, T_{n}\right] \rightarrow \mathbb{R}^{2}$ is a $C^{2}$ second order spiral spline satisfying $y_{\theta}^{(1)}\left(T_{0}\right)=V_{0}$ and $y_{\theta}^{(1)}\left(T_{n}\right)=V_{n}$. For $1 \leq j \leq n$ define $z_{j}: \mathbb{R}^{n} \times \mathbb{R}^{n} \rightarrow \mathbb{R}^{2}$ by

$$
z_{j}(u, v):=\int_{0}^{L_{j}}\left(\cos \theta_{j}(t), \sin \theta_{j}(t)\right) d t
$$

and define $z: \mathbb{R}^{n} \times \mathbb{R}^{n} \rightarrow\left(\mathbb{R}^{2}\right)^{n}$ by $z(u, v):=\left(z_{1}(u, v), z_{2}(u, v), \ldots, z_{n}(u, v)\right)$

Then $y_{\theta}$ interpolates $(Y, V)$ at $T$ precisely when $z_{j}(u, v)=Y_{j}-Y_{j-1}$ for all $1 \leq j \leq n$, namely when $(u, v) \in \mathbb{R}^{n} \times \mathbb{R}^{n}$ satisfies the nonlinear system of $2 n$ scalar equations in $2 n$ scalar unknowns

$$
z(u, v)=z^{*}:=\left(Y_{1}-Y_{0}, Y_{2}-Y_{1}, \ldots, Y_{n}-Y_{n-1}\right) \in\left(\mathbb{R}^{2}\right)^{n}
$$

whose right hand sides are found from $Y$. The key to solving such a system is a satisfactory initial guess $(\hat{u}, \hat{v})$ to a solution $(u, v)$. Set $\hat{u}=\hat{c}$ and $\hat{v}=\hat{d}$, where the $\hat{a}_{j}, \hat{b}_{j}, \hat{c}_{j}, \hat{d}_{j}$ are found in $\oint$. By Corollary $1, z(\hat{u}, \hat{v})=z^{*}+O\left(\epsilon^{5}\right)$.

In practice the integrals on the right hand side of $(39)$ are not easy to express as functions of $u, v$. So for numerical computations we replace the $z_{j}$ by approximations using the composite Simpson's Rule. Then $z$ becomes an explicit function of $(u, v)$ and 40$)$ is solved using Mathematica's FindRoot with $(\hat{u}, \hat{v})$ as an initial guess.

Example 4. Using composite Simpson with 6 intervals to estimate the $z_{j}$, FindRoot took around 0.3 seconds to obtain a numerical solution $\theta$ of (40) for the data in Example 1. Figure 4 shows the interpolant $y_{\theta}$ (yellow), together with the initial estimate $y_{\hat{\theta}}$ and data from Figure 1 .

Example 5. Perhaps it is no surprise that $y_{\theta}$ is successful for the data of Example 2, because $y_{\hat{\theta}}$ was already nearly interpolating. Figure 5 shows $y_{\theta}$ (yellow), together with $y_{\hat{\theta}}$ and the data. FindRoot took some 0.3 seconds to find $y_{\theta}$.

Example 6. ex6 More impressively, it takes some 0.3 seconds to improve $y_{\hat{\theta}}$ in Example 3 to the interpolant $y_{\theta}$ shown (yellow) in Figure 6. Whereas $y_{\hat{\theta}}$ comprehensively failed to interpolate, the $C^{1}$ cubic spline $\hat{\theta}:\left[T_{0}, T_{n}\right] \rightarrow \mathbb{R}$ retains considerable informative power. This power, masked to some degre ${ }^{12}$ by the second order spiral spline $y_{\hat{\theta}}:\left[T_{0}, T_{n}\right] \rightarrow \mathbb{R}^{2}$, is key to the method of the present section $\$ 10$

\section{CONCLUSION}

Given interpolation data $(Y, V)$ for convex unit-speed $C^{2}$ curves in $\mathbb{R}^{2}$, relative to some sequence $T$, we develop a method for interpolating $(Y, V)$ at $T$ by a $C^{2}$ second order spiral spline $y_{\theta}:\left[T_{0}, T_{n}\right] \rightarrow \mathbb{R}^{2}$. The method is developed using asymptotic arguments to define a pair of tridiagonal linear systems whose solutions define a real-valued $C^{1}$ cubic polynomial spline $\hat{\theta}$ with prescribed values at $T_{0}, T_{n}$. For sufficiently finely sampled data, $\hat{\theta}$ already defines a $C^{2}$ second order spiral spline $y_{\hat{\theta}}$ that approximately interpolates $(Y, V)$ at $T$. More importantly, $\hat{\theta}$ is used to start a numerical method that finds another $C^{1}$ cubic polynomial spline $\theta$. Then $y_{\theta}$ is the desired $C^{2}$ second order interpolating spiral spline.

\section{REFERENCES}

[1] Di Antonio L. The fabric of the universe is most perfect: Euler's research on elastic curves, in Euler at, 300: an appreciation, 239-260, Mathematical Association of America (2007).

[2] Audoly, B., Pomeau, Yves, Elasticity and Geometry: From Hair Curls to the Non-linear Response of Shells, Oxford University Press (2010).

[3] Baran, Ilya, Lehtinen, Jaako and Popovic, Jovan, Sketching Clothoid Splines Using Shortest Paths, Eurographics 0 (1981).

[4] Florence Bertails-Descoubes, Super-Clothoids, Eurographics 31 (2012)

[5] G. Birkhoff, H. Burchard and D. Thomas, Nonlinear Interpolation by Splines, Pseudosplines and Elastica, General Motors Research Laboratories Report 468, (1965), 1-13.

[6] Bruckstein, Alfred M., Holta Robert, J., and Netravalia, Arun, N., Discrete elastica, Applicable Analysis 78 (2001) $453-485$.

[7] Brunnett, Guido and Wendt, Jörg, A Univariate Method for Plane Elastic Curves, Computer Aided Geometric Design 14 (1997) $273-292$.

[8] Coope, Ian, D., Curve Fitting With Nonlinear Spiral Splines, Department of Mathematics, University of Canterbury NZ, No 63 (1991), 1-14.

[9] Dai, Ran and Cochran, John E. Jr. (2010) Path Planning and State Estimation for Unmanned Aerial Vehicles in Hostile Environments, Journa of Guidance, Control and Dynamics 33 (2), 595-601.

10] de Boor, Carl, A Practical Guide to Splines, Applied mathematical Sciences 27, Springer (1978).

11] Eberly, David, Moving Along a Curve with Specified Speed, http://www.geometrictools.com/ (2007) 1-15.

[12] Edwards, J.A., Exact equations of the nonlinear spline, ACM Transactions on Mathematical Software 18 (1992), 174-192

[13] Golomb, Michael and Jerome, Joseph, Equilibria of the Curvature Functional and Manifolds of Interpolating Spline Curves, SIAM J. on Mathematical Analysis 13 (3) (1982), 412-458.

[14] Alonzo Kelly and Brian Nagy, Reactive Nonholonomic Trajectory Generation via Parametric Optimal Control, International Journal of Robotics Research 22 (2003), 583-601.

[15] Levien, Raph, The Elastica: a Mathematical History, Technical Report No. UCB/EECS-2008-103 http://ww. eecs.berkeley.edu/Pubs/TechRpts/2008/EECS-2008-103.html August 23, 2008.

[16] E.H. Lee and G.E. Forsythe, Variational Study of Nonlinear Spline Curves, SIAM Review 15 (1973), 120-133.

[17] Linners, Anders, Unified Representations of Nonlinear Splines, Journal of Approximation Theory 84 (1996), 315-350.

[18] Looker, Jason R., Constant Speed Interpolating Paths, DSTO Defence Science and Technology Organisation, AR 014-939. DSTO-TN-0989, March 2011

[19] D.S. Meek and R.S.D. Thomas, A Guided Clothoid Spline, Computer Aided Geometric Design 8 (1991) 163-174.

[20] D.S. Meek and D.J. Walton, An Arc Spline Approximation to a Clothoid, Journal of Computational and Applied Mathematics 170 (2004), 59-77.

[21] Peterson, John W., Arc-Length Parameterization of Spline Curves http://www.saccade.com/writing/graphics/RE-PARAM.PDF

${ }^{12}$ Interpolation errors accumulate along the trajectory. The related curve $\bar{y}_{\hat{\theta}}$ of $\$ 5$ avoids this defect, and is better for diagnostics, but fails to be continuous at interpolation points. 


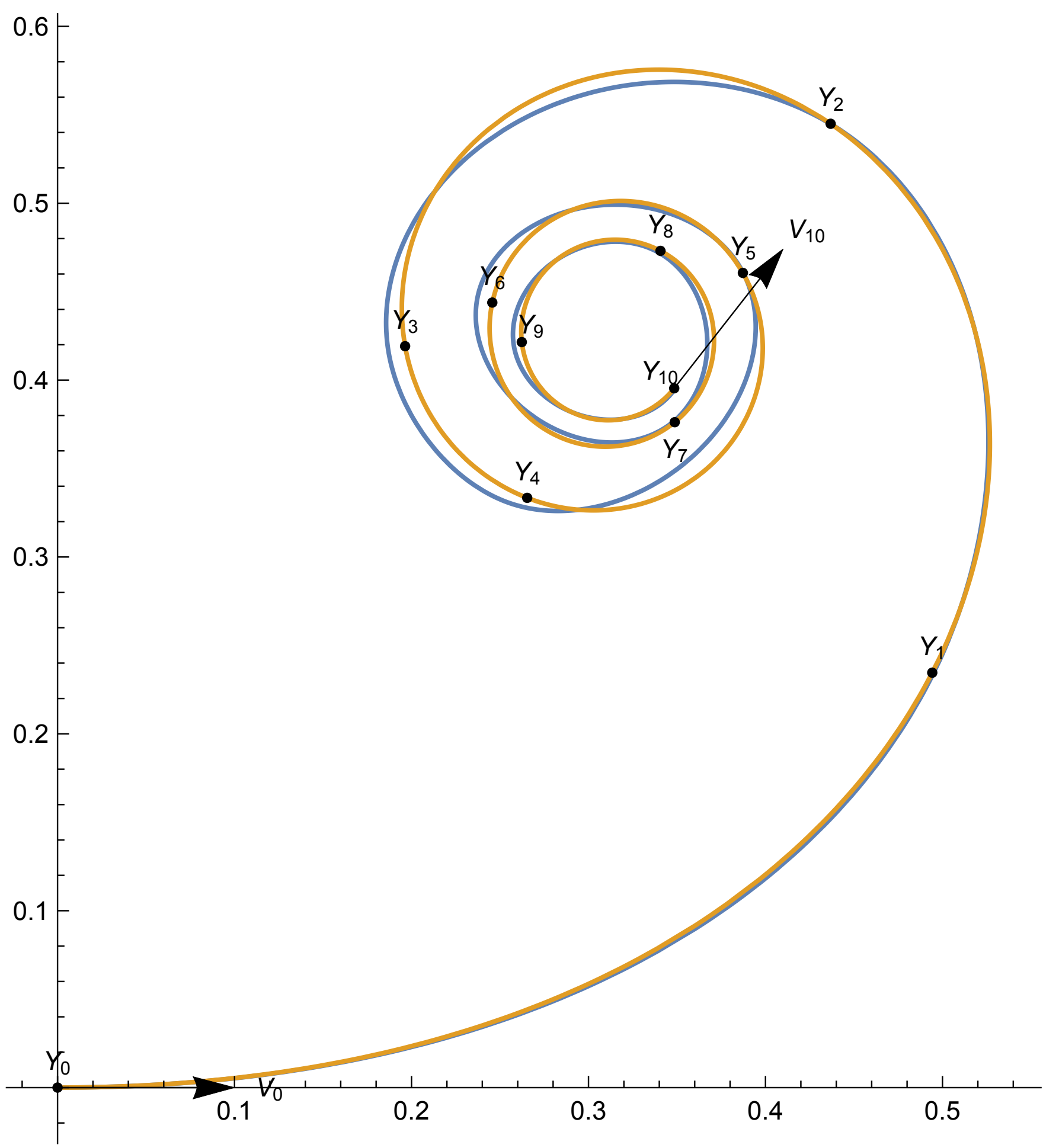

FiguRe 4. $y_{\theta}$ (yellow) and $y_{\hat{\theta}}$ for Example 4

[22] Singer, David A., Lectures on Elastic Curves and Rods, in AIP Conference Proceedings 1002, Curvature and Variational Modelling in Physics and Biophysics, Santiago de Compostela, Spain, 17-18 September 2007 (eds Oscar J. Garay, Eduardo Garcia-Río, Ramón Vázquez-Lorenzo), $3-32$.

[23] Stoer, Josef, Curve Fitting With Clothoidal Splines, J. Research of the National Bureau of Standards 87 No. 4 (1982) 318-346.

[24] Arc-Length Parameterized Spline Curves for Real-Time Simulation, Wang, Hongling, Keaney, Joseph, Atkinson, Kendall, Curve and Surface Design: Saint Malo (2002) Lyche, T., Mazure, M.-L., Schumacher, L. (eds) 387-396.

Lyle.Noakes@uwa.edu.au (Department of Mathematics \& Statistics, The University of Western Australia, 35 Stirling Highway, Crawley, WA 6009, AUSTRALIA) 


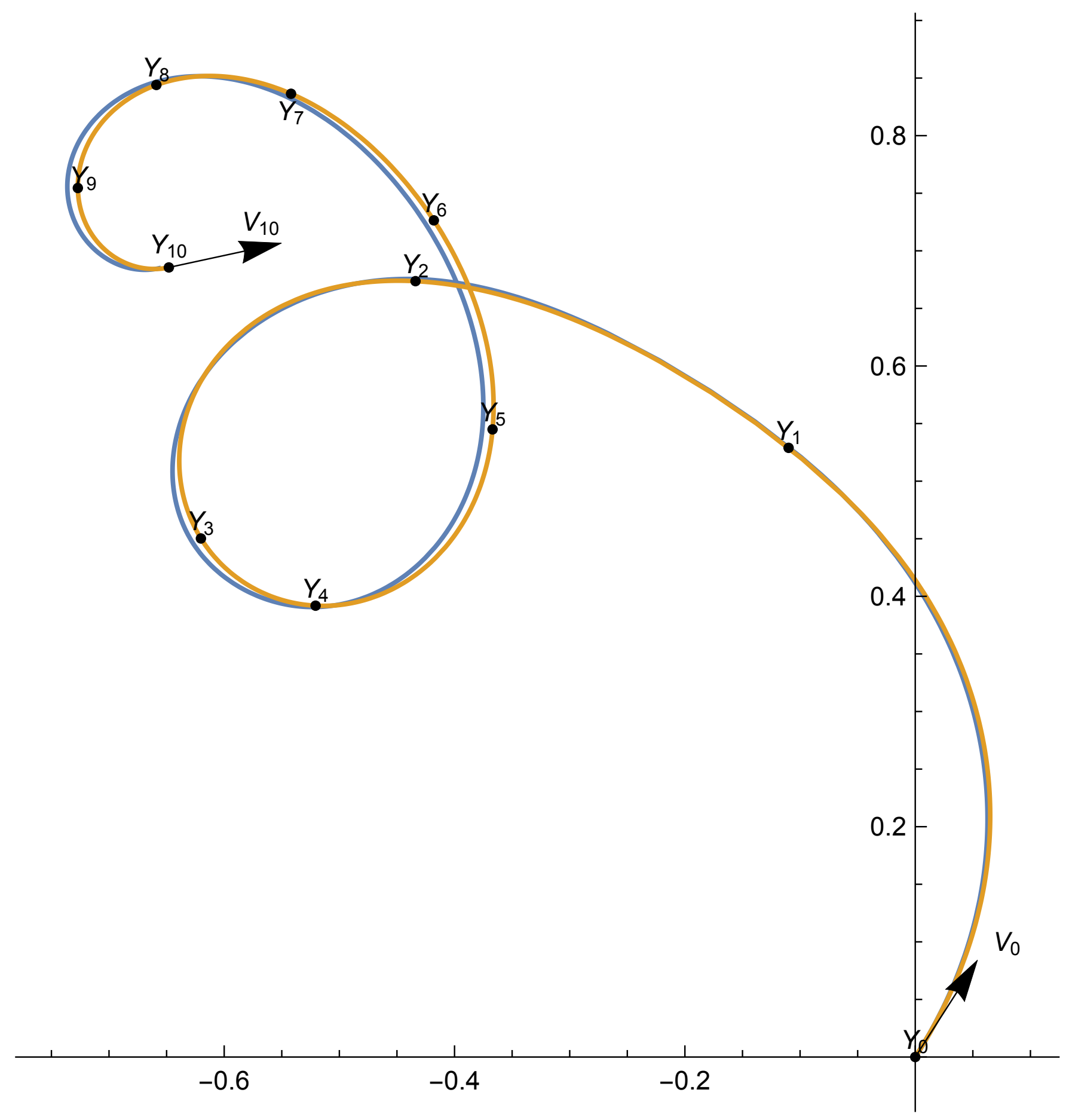

Figure 5. $y_{\theta}$ (yellow) and $y_{\hat{\theta}}$ in Example 5 


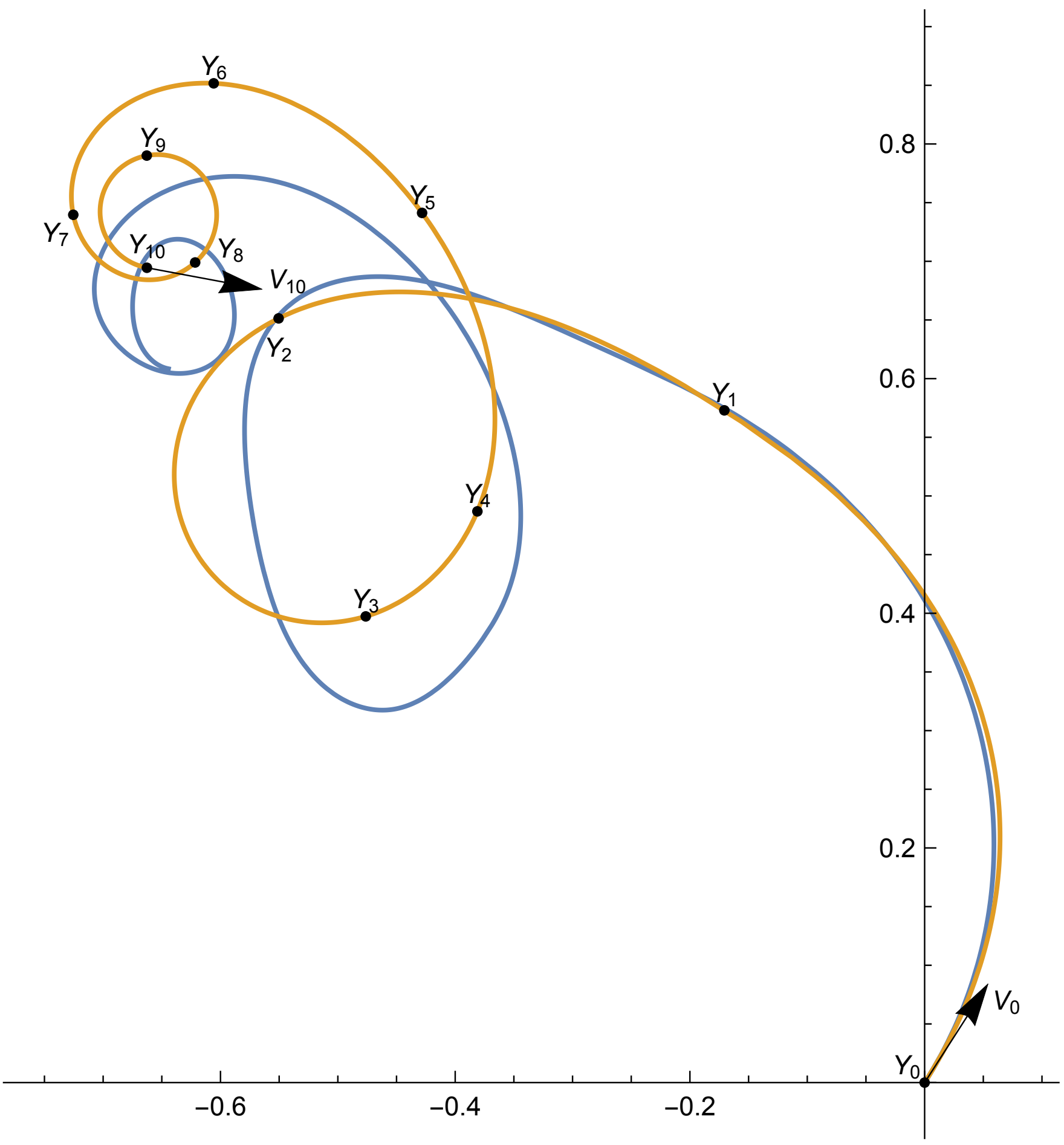

Figure 6. $y_{\theta}$ (yellow) and $y_{\hat{\theta}}$ for Example 6 